\title{
Lifetime and Leptonic Branching Ratios of the Tau Lepton
}

\author{
M. McCubbin*
}

\begin{abstract}
This paper describes recent measurements of the tau lifetime and leptonic branching rations, concentrating on results from DELPHI at LEP but also mentioning results from other experiments, in particular those from other LEP experiments. The DELPHI analysis is based on around $6500 \tau+\tau$-pairs in the barrel region, taken in 1991. The combined LEP results show that the ratio of the tau to muon Fermi coupling constants is consistant with unity.
\end{abstract}




\title{
LIFETIME AND LEPTONIC BRANCHING RATIOS \\ OF THE TAU LEPTON
}

\author{
Martin McCubbin \\ Department of Physics \\ Oliver Lodge Laboratory \\ University of Liverpool \\ Liverpool, England
}

\begin{abstract}
This paper describes recent measurements of the tau lifetime and leptonic branching ratios, concentrating on results from DELPHI at LEP but also mentioning results from other experiments, in particular those from other LEP experiments. The DELPHI analysis is based on around $6500 \tau^{+} \tau^{-}$pairs in the barrel region, taken in 1991. The combined LEP results show that the ratio of the tau to muon Fermi coupling constants is consistant with unity.
\end{abstract}

\section{INTRODUCTION}

The Standard Model gives a prediction for the tau lifetime $e^{1,2}$ :

$$
\tau_{\tau}=\tau_{\mu}\left(\frac{G_{\mu}}{G_{\tau}}\right)^{2}\left(\frac{m_{\mu}}{m_{\tau}}\right)^{5} B R(\tau \rightarrow e \nu \nu) .
$$

Assuming $G_{\tau}=G_{\mu}$ and using the world average values ${ }^{3}$ for $B R(\tau \rightarrow e \nu \nu), \tau_{\mu}, m_{\mu}$ and $m_{\tau}$, equation (1) gives

$$
\tau_{\tau}=286 \pm 4 f s,
$$

which is $2 \sigma$ away from the world average tau lifetime ${ }^{3}$

$$
\tau_{\tau}=305 \pm 6 f s .
$$

Further measurements of the tau lifetime are needed to resolve or confirm this discrepancy, or alternatively to test $\tau-\mu$ universality.

\section{THE DELPHI DETECTOR}

The DELPHI detector has been described in detail elsewhere ${ }^{-4}$. The microvertex detector ${ }^{5}$, vital for the lifetime measurement, comprises 3 layers of single-sided silicon detectors arranged cylindrically around the interaction point at radii of $6 \mathrm{~cm}, 9 \mathrm{~cm}$ and $11 \mathrm{~cm}$. The $50 \mu \mathrm{m}$ readout pitch gives an intrinsic r- $\phi$ resolution of $6 \mu \mathrm{m}$.

The track extrapolation resolution at the vertex is found to be

$$
\sigma=\sqrt{26^{2}+\left(\frac{65}{p_{t}(G e V / c)}\right)^{2}} \mu m,
$$

i.e. approximately $30 \mu \mathrm{m}$ for tau decays.

The centre of the interaction region was determined every $100 Z^{0} \rightarrow q \bar{q}$ events, with a precision of $10 \mu \mathrm{m}$. This was taken as the $\tau^{+} \tau^{-}$production point. The extent of the interaction region is given by $\sigma_{x}=145 \mu m$ and $\sigma_{y}=7 \mu m$, where $\mathrm{x}$ and $\mathrm{y}$ are the horizontal and vertical directions in the plane transverse to the beam, respectively.

\section{TAU LIFETIME}

DELPHI uses four methods for measuring the tau lifetime, which will be reviewed in this section. Results from the other LEP experiments are also mentioned. 


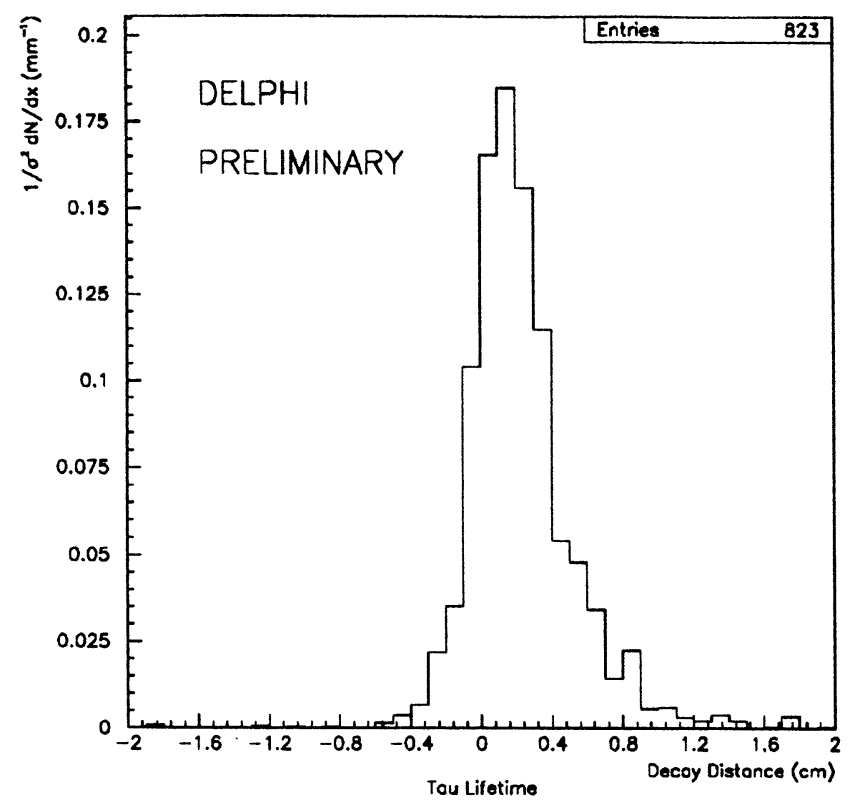

Figure 1. The 3-prong decay distance distribution (DELPHI).

\section{Vertex Method}

This method ${ }^{6}$ involves looking at $\tau^{+} \tau^{-}$ events which decay into a 1 versus 3 chargedtrack topology, reconstructing the decay vertex on the 3-prong side, and determining the decay distance from the production point. After event selection, track and vertex quality cuts, 823 secondary vertices are found. The decay distance distribution is shown in figure 1. A maximum likelihood fit to the data gives

$$
\tau_{\tau}=303 \pm 13(\text { stat }) \pm 7(\text { sys }) f s
$$

Other recent measurements of the tau lifetime at $\operatorname{LEP}^{9,13,14}$ using this method are shown in figure 2.

\section{Impact Parameter Method}

For this method ${ }^{6}, \tau^{+} \tau^{-}$events are selected which decay into 1 versus 1 chargedtrack topologies. The impact parameter ${ }^{-1}$ for 1 -prongs is related to the tau lifetime, and is signed using the charged track in the opposite

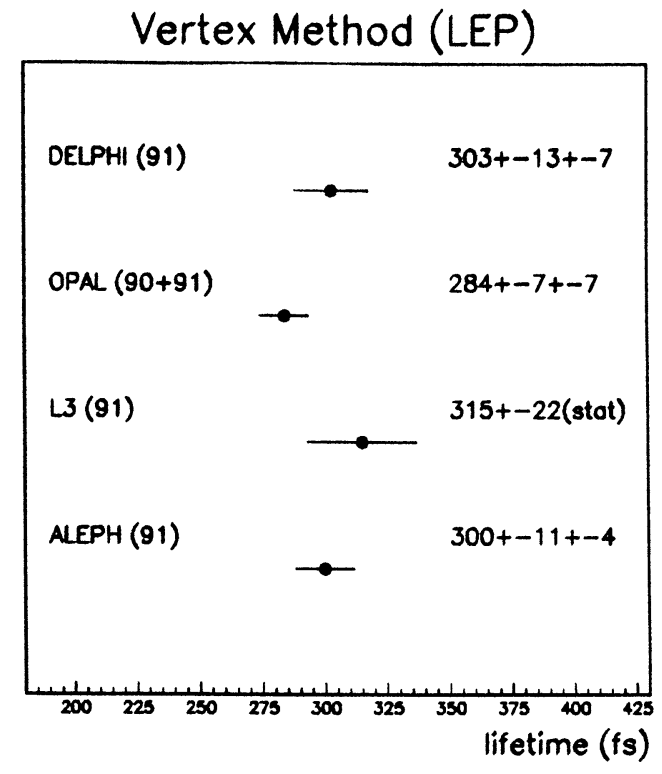

Figure 2. Recent LEP tau lifetime numbers (vertex method).

hemisphere to define the tau direction. After event selection and track quality cuts there are 6113 1-prong tau decays. The signed impact parameter distribution is shown in figure 3 . A maximum likelihood fit to the data gives

$$
\tau_{\tau}=304 \pm 11 \pm 6 f s
$$

Some recent measurements of the tau lifetime at $\operatorname{LEP}^{10,13,14}$ using this method are shown in figure 4.

\section{Decay Angle Correlation Method}

Again, $\tau^{+} \tau^{-}$events decaying into 1 versus 1 charged-track topologies are used. This method $^{7}$ relies on the fact that the difference in impact parameters for the two tracks is proportional to the projected acoplanarity, with a constant of proportionality related to the lifetime. In addition to the event selection made for the impact parameter method, cuts are made in order to reject radiative events. After cuts, 2873 events remain in the projected acoplanarity range -0.2 to 0.2 (radians). The mean difference in impact parameter versus the projected acoplanarity is shown in figure 


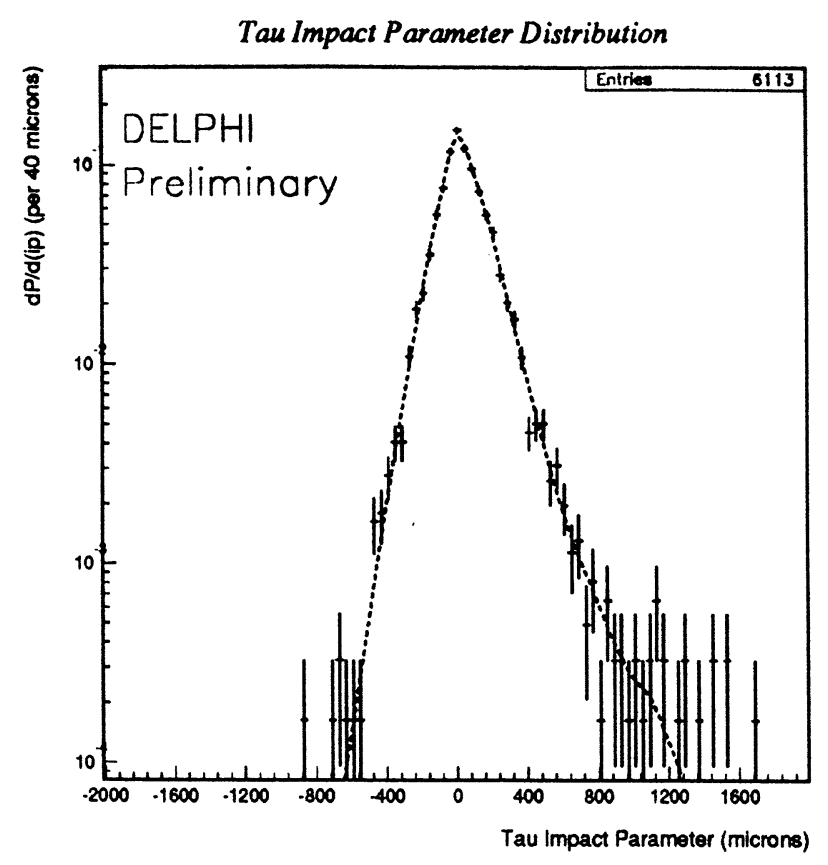

Figure 3. The signed impact parameter distribution with fit (DELPHI).

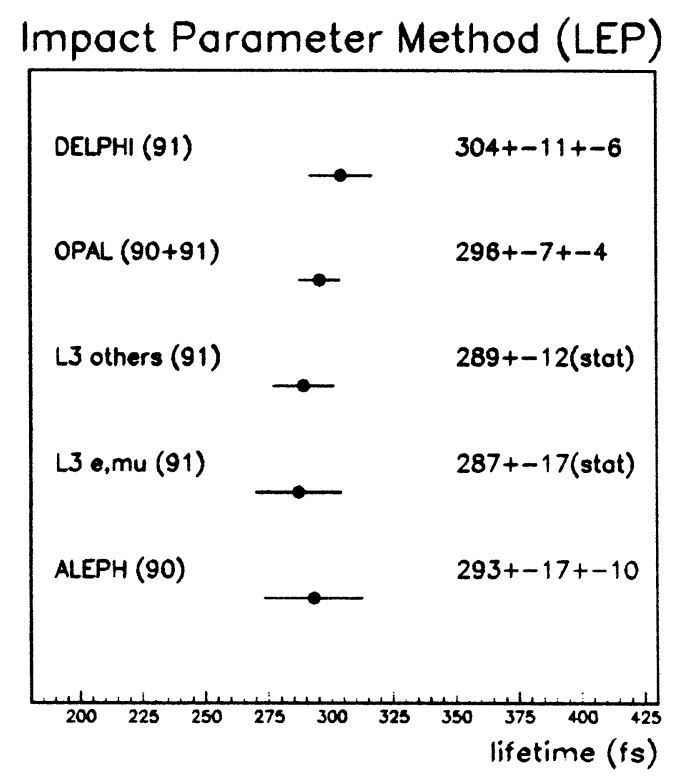

Figure 4. Recent LEP tau lifetime numbers (impact parameter method).

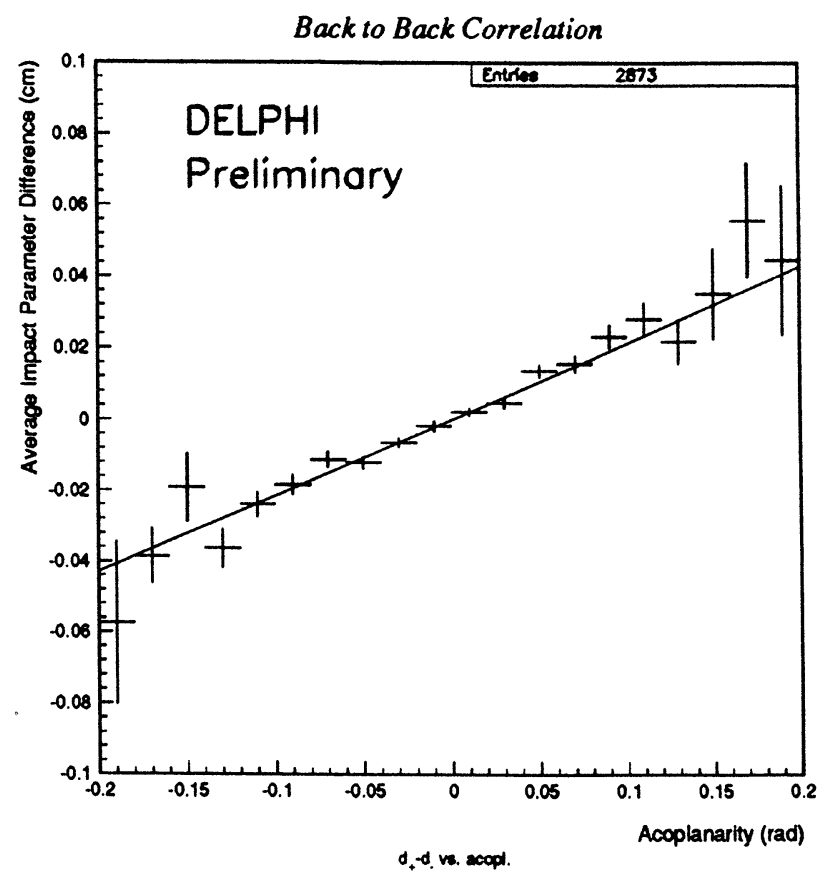

Figure 5. Mean impact parameter difference versus projected acoplanarity with fit (DELPHI).

5. A straight line fit to the data, including the effect of biases due to the remaining radiative events, gives

$$
\tau_{\tau}=299 \pm 11 \pm 6 f s .
$$

ALEPH has carried out a measurement of the tau lifetime using this method for their 1991 data $^{11}$, with the result

$$
\tau_{\tau}=311 \pm 12 \pm 4 f s
$$

\section{Missed Distance Method}

Here the sum of the impact parameters for events in which $\tau^{+} \tau^{-}$decays into 1 versus 1 charged-track topologies is used to extract the tau lifetime ${ }^{8}$. The same data sample as in the impact parameter difference method is used, together with extra track quality cuts; this leaves 2353 events. The distribution of impact parameter sum, or missed distance, is shown in figure 6. A log likelihood fit to the data gives

$$
\tau_{\tau}=301 \pm 9 \pm 6 f s
$$




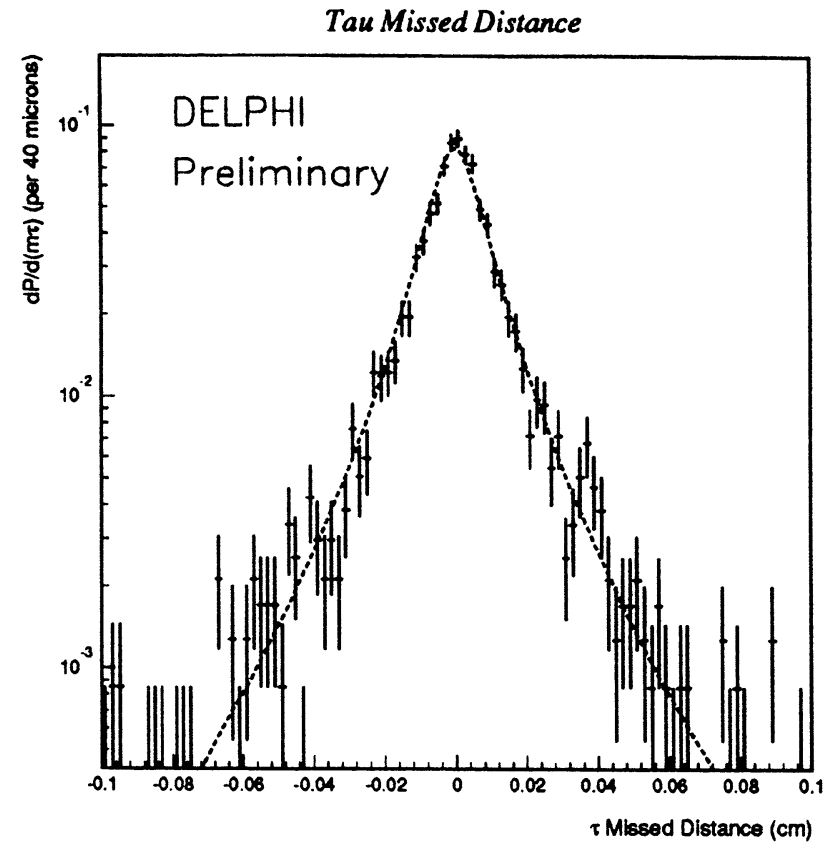

Figure 6. The missed distance distribution with fit (DELPHI).

ALEPH has carried out a measurement of the tau lifetime using a similar method on their 1991 data sample ${ }^{12}$, and obtains the result

$$
\tau_{\tau}=287 \pm 8 \pm 5 f s
$$

\section{Summary of Tau Lifetime Results}

Figure 7 shows a summary of recent measurements of the tau lifetime $\mathrm{e}^{6-15}$; the numbers quoted for each LEP experiment are derived by combining the results for each method used by that experiment.

\section{TAU LEPTONIC BRANCHING RATIOS}

The 1991 DELPHI $\tau^{+} \tau^{-}$event sample for the measurement of $B R(\tau \rightarrow e \nu \nu)$ and $B R(\tau \rightarrow \mu \nu \nu)$ comprises around 6000 events, from which $1272 \tau \rightarrow e \nu \nu$ decays are selected with an efficiency of $60 \%$, and $1579 \tau \rightarrow \mu \nu \nu$ decays are selected with an efficiency of $72 \%$. The results for the leptonic branching ratios

\section{Tau Lifetime}

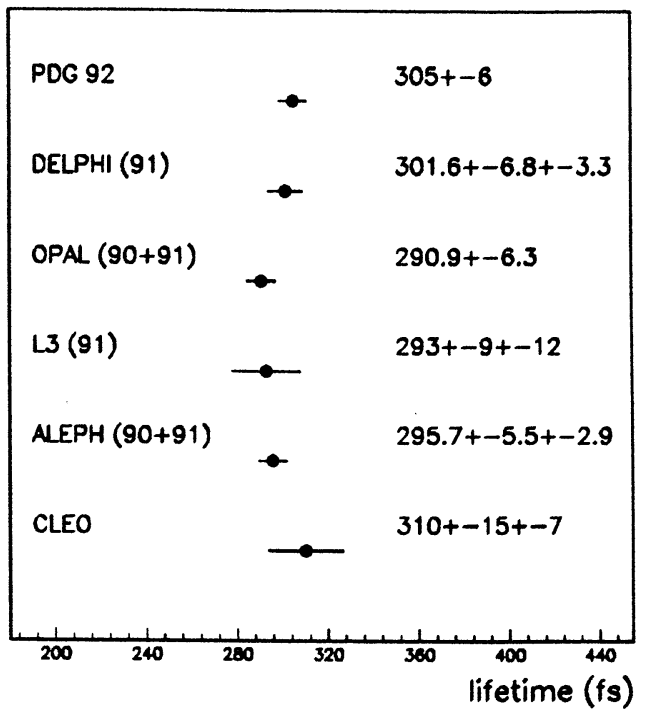

Figure 7. Summary of recent tau lifetime results.

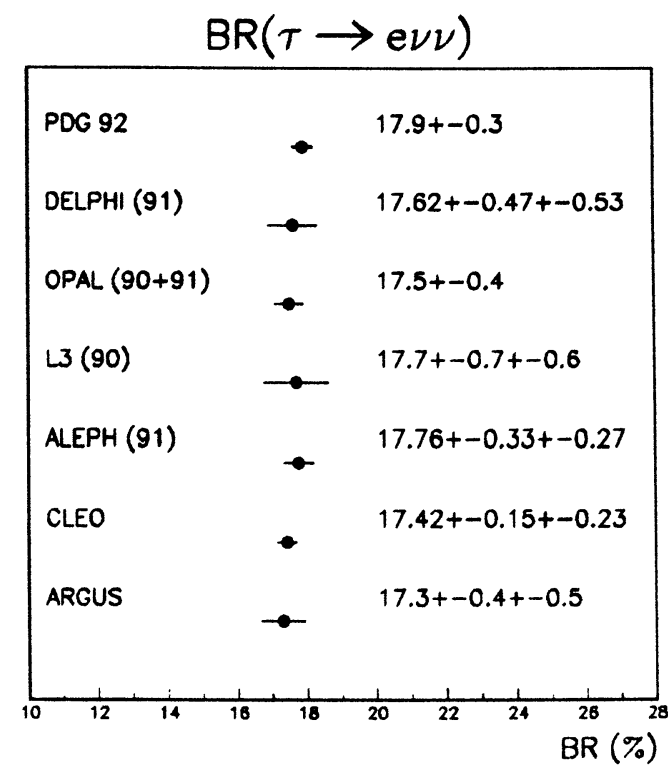

Figure 8. Summary of recent results on the electronic branching ratio. 


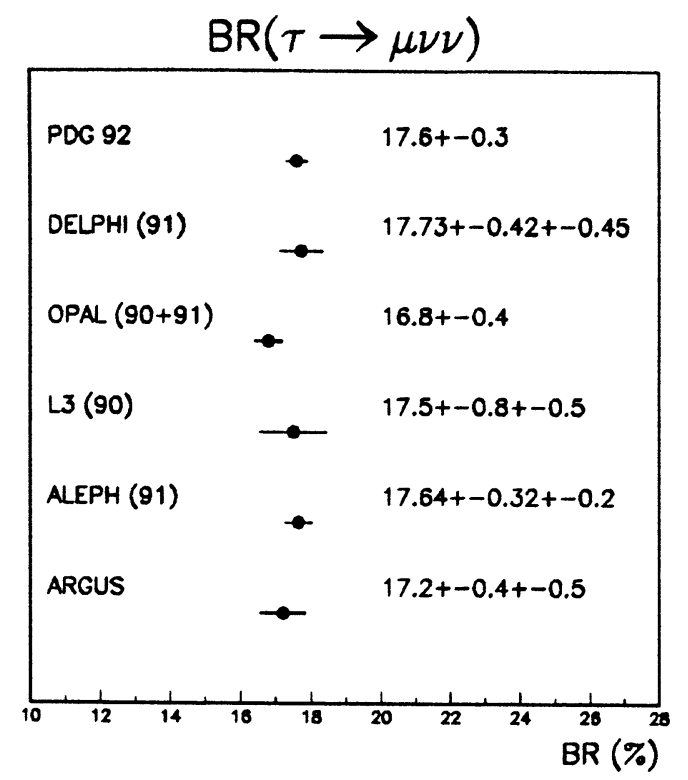

Figure 9. Summary of recent results on the muonic branching ratio.

are

$$
\begin{aligned}
& B R(\tau \rightarrow e \nu \nu)=17.62 \pm 0.47 \pm 0.53 \%, \\
& B R(\tau \rightarrow \mu \nu \nu)=17.73 \pm 0.42 \pm 0.45 \% .
\end{aligned}
$$

Figures 8 and 9 show the comparison of these numbers with other recent leptonic branching ratio results ${ }^{16-21}$.

\section{CONCLUSIONS}

Averaging the LEP values, not taking into account common systematic errors, yields

$$
\begin{gathered}
B R(\tau \rightarrow e \nu \nu)=17.63 \pm 0.26 \% \\
B R(\tau \rightarrow \mu \nu \nu)=17.34 \pm 0.24 \% \\
\tau_{\tau}=295.3 \pm 3.7 f s .
\end{gathered}
$$

Using these values, and a value for the tau mass $m_{\tau}=1777.1 \pm 0.5 \mathrm{MeV} / \mathrm{c}^{2}$, derived by averaging the measurements from three different experiments ${ }^{20,22,23}$, equation (1) gives

$$
\frac{G_{\tau}}{G_{\mu}}=0.987 \pm 0.010
$$

which is a consistant with unity. Including the ARGUS and CLEO values mentioned in this paper for $B R(\tau \rightarrow e \nu \nu)$ and the tau lifetime, we get

$$
\frac{G_{\tau}}{G_{\mu}}=0.983 \pm 0.008
$$

\section{REFERENCES}

1. Y.S.Tsai, "Decay correlations of heavy leptons in $\mathrm{e}^{+} \mathrm{e}^{-} \rightarrow 1^{+} 1^{-}$, Phys. Rev. D4, pp. 2821-2837, (1971).

2. H.B.Thacker and J.J.Sakurai, "Lifetimes and branching ratios of heavy leptons", Phys. Lett. 36B, pp. 103-105, (1971).

3. Particle Data Group, M.Aguilar-Benitez et al., Phys. Rev. D45, part 2, (1992).

4. DELPHI collaboration, "The DELPHI detector at LEP", Nucl. Instr. and Meth. A303, pp. 233-276, (1991).

5. "DELPHI microvertex detector", submitted to this conference and Nucl. Instr. and Meth.

6. "A measurement of the tau lifetime", DELPHI collaboration, submitted to this conference.

7. "A measurement of the tau lifetime using the impact parameter difference method", DELPHI internal note 92-131 PHYS 231, (1992).

8. "A measurement of the tau lifetime using the miss distance method", DELPHI internal note 92-132 PHYS 232, (1992).

9. "Tau lifetime with the decay length method using VDET", ALEPH internal note 92-22, (1992).

10. ALEPH collaboration, Decamp et al., "Measurement of the tau lepton lifetime", Phys. Lett. 279B, pp. 411-421, (1992).

11. "Measurement of the tau lifetime using the impact parameter difference method: 1991 data with VDET", ALEPH internal note $92-73,(1992)$. 
12. "Using the sum of impact parameters to measure the tau lifetime", ALEPH internal note 92-27, (1992).

13. OPAL collaboration, private communication.

14. L3 collaboration, private communication.

15. Stroynowski, "Tau Decay at CLEO", Proceedings of the LP-HEP '91, page 562, (1991).

16. "A measurement of $\tau \rightarrow e \nu \nu$ branching ratio with 1991 data", ALEPH internal note 92-94, (1992).

17. "A measurement of $\tau \rightarrow \mu \nu \nu$ branching ratio with 1991 data", ALEPH internal note 92-57, (1992).

18. OPAL collaboration, private communication.

19. L3 Collaboration, Adeva et al., "Decay properties of tau leptons measured at the $\mathrm{Z}^{0}$ resonance", Phys. Lett. 265B, pp. 451461, (1991).

20. "New results on tau lepton from CLEO", submitted to this conference.

21. ARGUS collaboration, Albrecht et al., "Measurement of exclusive one-prong and inclusive three-prong branching ratios of the $\tau$ lepton", Zeit. Phys. C53, pp. 367374, (1992).

22. "Tau physics at Argus", submitted to this conference.

23. "Measurement of the mass of the tau lepton", BES collaboration, submitted to this conference. 